\title{
A clinical study of Noonan syndrome
}

\author{
M Sharland, M Burch, W M McKenna, M A Paton
}

\begin{abstract}
Clinical details are presented on 151 individuals with Noonan syndrome (83 males and 68 females, mean age 12.6 years). Polyhydramnios complicated $33 \%$ of affected pregnancies. The commonest cardiac lesions were pulmonary stenosis $(62 \%)$, and hypertrophic cardiomyopathy $(20 \%)$, with a normal echocardiogram present in only $12.5 \%$ of all cases. Significant feeding difficulties during infancy were present in $76 \%$ of the group. Although the children were short $(50 \%$ with a height $<3$ rd centile), and underweight (43\% with a weight $<3$ rd centile), the mean head circumference of the group was on the 50th centile. Motor milestone delay was usual, the cohort having a mean age of sitting unsupported of 10 months and walking of 21 months. Abnormal vision (94\%) and hearing $(40 \%)$ were frequent findings, but $89 \%$ of the group were attending normal primary or secondary schools. Other associations included undescended testicles (77\%), hepatosplenomegaly $(50 \%)$, and evidence of abnormal bleeding ( $56 \%$ ). The mean age at diagnosis of Noonan syndrome in this group was 9.0 years. Earlier diagnosis of this common condition would aid both clinical management and genetic counselling.
\end{abstract}

Nine patients with a characteristic facies, valvular pulmonary stenosis, and short stature were first reported by Dr Jacqueline Noonan in 1963. ${ }^{1}$ Her subsequent paper described 19 patients (12 males and seven females) with a typical facies, congenital heart disease, and clinical features of Turner's syndrome with normal chromosomes. ${ }^{2}$ These papers ended an era of confused terminology surrounding the individual with a Turner phenotype. Turner originally reported a series of older women with webbed neck, short stature, cubitus valgus, and sexual infantilism in $1938 .{ }^{3}$ Ullrich had independently recognised both males and females with a similar phenotype which he named status Bonnevie Ullrich, after Bonnevie's work on mice. ${ }^{4}$ It was only after Ford et al had identified the karyotype $45 \mathrm{X}$ in a patient with the diagnosis of Turner's syndrome that a clear division was made between Turner's syndrome $(45 \mathrm{X}$, female only) and Noonan syndrome (normal karyotype, equal sex incidence). ${ }^{5}$ Considerable differences between the two disorders are now well documented.

Recent excellent reviews of Noonan syndrome have been produced and have commented on areas of limited knowledge. ${ }^{67}$ These included difficulty in making the clinical diagnosis, the lack of a diagnostic biochemical marker, no clear advice about management or outcome, and the absence of simple recurrence risks in genetic counselling. This study was undertaken in an attempt to answer some of these questions. A further aim was to identify pedigrees suitable for linkage analysis, in an attempt to map the gene for Noonan syndrome.

\section{Methods}

Consultant paediatricians, paediatric cardiologists, and clinical geneticists in the UK were contacted and asked for names of patients under their care with Noonan syndrome. Permission to approach over 400 individuals was obtained. The first 200 patients ascertained were then contacted and invited to participate in the research. If the family was willing to help with the project, all first degree relatives were asked to visit St George's Hospital for one day. Travelling expenses were paid if necessary in an attempt to remove a social class bias. The ascertained proband and all first degree relatives underwent a standardised symptom review and clinical examination. Echocardiography and clinical photography were performed on all the family members. Blood was taken for DNA studies. Further specific examinations were performed on different cohorts during the project. These included abdominal ultrasound, orthoptic and ophthalmological examination, coagulation studies, and speech therapy assessment.

\section{Results}

A clinical diagnosis of Noonan syndrome was made in a total of 151 individuals $(83$ males and 68 females) derived from 123 families. The age range of the patients was from one week to 60 years (mean 12.6 years). Data were obtained from family questionnaires, clinical examination, and specific investigations.

\section{OBSTETRIC DATA}

The mean gestational age at delivery was 39 weeks. A clinical diagnosis of polyhydramnios had been made in $\mathbf{4 3}$ of the 130 pregnancies whose obstetric details were available (33\%). The mode of delivery had been ascertained in 149 pregnancies. A normal vaginal delivery occurred in 103 cases $(69 \%)$, a forceps delivery was performed in 13 cases $(9 \%)$, and a caesarean section in 32 cases $(21 \%)$. The birth weight was available for 114 babies born at term (38-42 
weeks) and had a mean (SD) of 3450 (510) g. The majority of parents had recognised that their baby was generally oedematous at birth, and lost excessive weight over the first week of life, but this was not possible to quantify.

\section{CARDIAC DATA}

Corrective cardiac surgery had been performed on 31 individuals at the time of the project. All 151 probands had echocardiograms performed. The following data are also derived from echocardiograms performed by regional cardiac units before corrective surgery. Pulmonary stenosis was detected in 93 cases (62\%) and was usually due to a dysplastic pulmonary valve. The pulmonary stenosis was an isolated lesion in 65 cases (43\%), associated with an atrial, ventricular, or atrioventricular septal defect in 20 cases (13\%), and with hypertrophic cardiomyopathy in a further eight cases $(6 \%)$. Hypertrophic cardiomyopathy, including asymmetrical septal hypertrophy, was present in $20 \%$ of the study group. It was found as an isolated lesion in 19 cases (13\%), and in two cases was associated with a patent ductus arteriosus. Three children had undergone surgical myomectomy, and two further children had required cardiac transplantation for severe heart failure in infancy. Other cardiac findings included isolated septal defects (nine cases) and mitral valve prolapse (four cases). Only 19 individuals $(12 \cdot 5 \%)$ had normal echocardiograms.

\section{FACIAL CHANGES}

The predominant facial features of Noonan syndrome seen in this group were hypertelorism, down slanting palpebral fissures with high arched eyebrows, epicanthic folds, full upper lip, depressed nasal root with a wide nasal base, and low set ears with a thickened helix. Severe ptosis (either unilateral or bilateral) was present in 63 individuals $(42 \%)$. Obvious webbing of the skin of the neck (excluding the common apparent neck shortening) was present in only 34 cases $(23 \%)$. The characteristic facial changes of children with Noonan syndrome at varying ages are demonstrated in fig 1,2 , and 3.

\section{FEEDING DIFFICULTIES}

Feeding histories were obtained in 144 cases. There were no significant feeding difficulties in 35 cases (24\%). Mild feeding problems (defined as the infant having a poor suck, with each feed taking on average over an hour to complete) were reported in 21 cases (15\%). Moderate feeding difficulties (defined as a very poor suck, with slow feeding and recurrent vomiting with most feeds) were reported in 54 cases (38\%). Severe feeding problems (defined as requiring tube feeding for two weeks or longer in a term infant) were found in 34 cases (24\%).

DEVELOPMENTAL DATA

The age at which the child first sat unsupported was known in 126 cases, and had a mean (SD) age of $10(4.5)$ months. The time of first

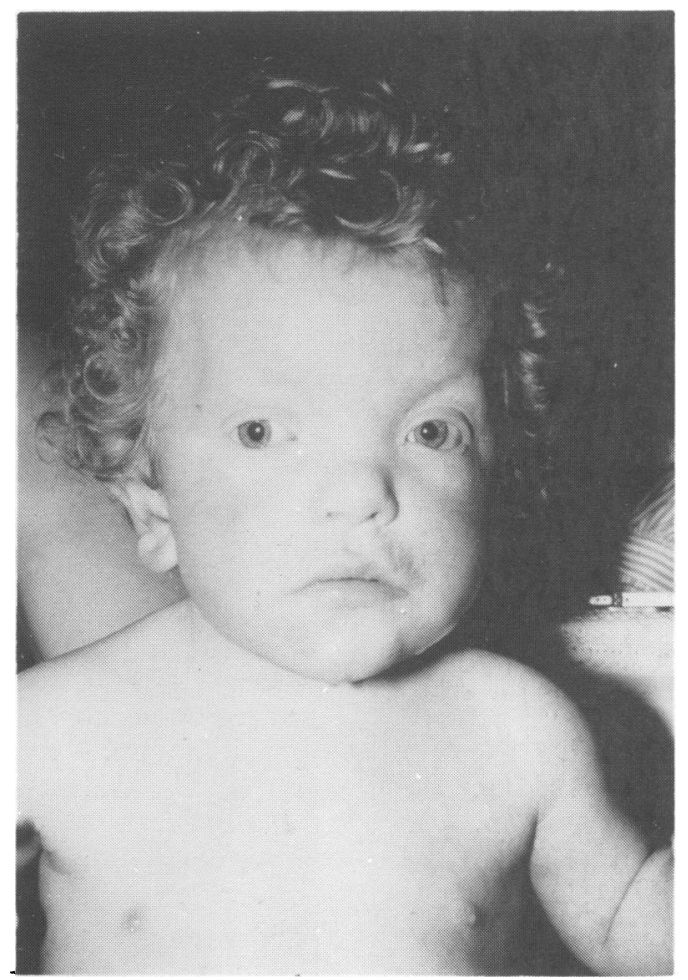

Figure 1 Child with Noonan syndrome aged 2 years.

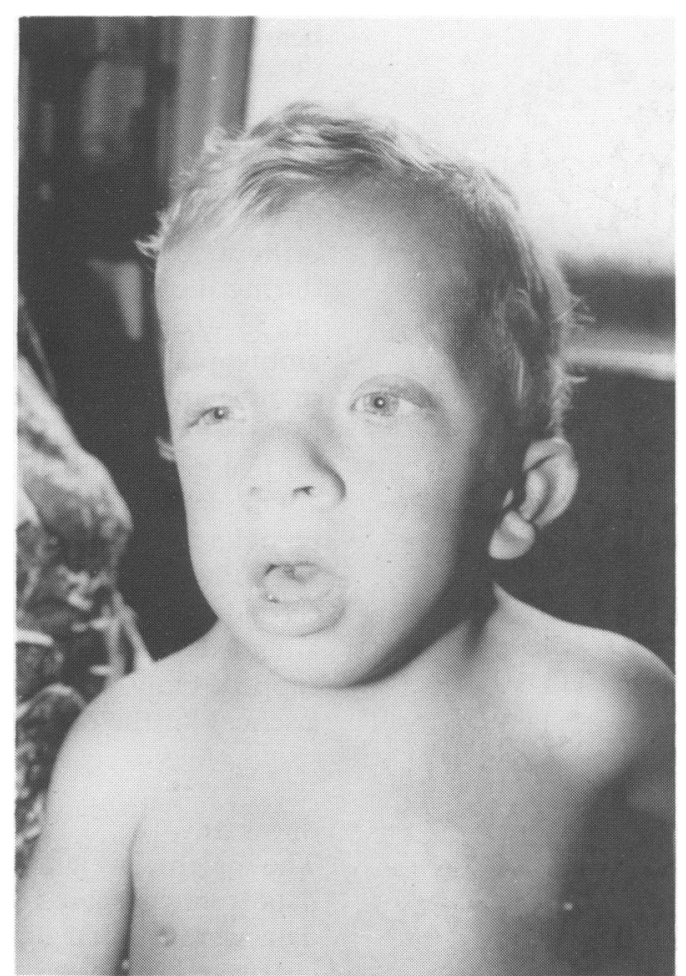

Figure 2 Child with Noonan syndrome aged 4 years.

walking unsupported was available for 112 cases, and had a mean (SD) age of $21(10 \cdot 2)$ months. The age at which the child could first talk in simple two word sentences was known in 102 children, and had a mean (SD) age of 31 (9.6) months.

Hearing tests had been performed on 146 of the group and hearing loss was reported in 58 $(40 \%)$. In the great majority this was due to 


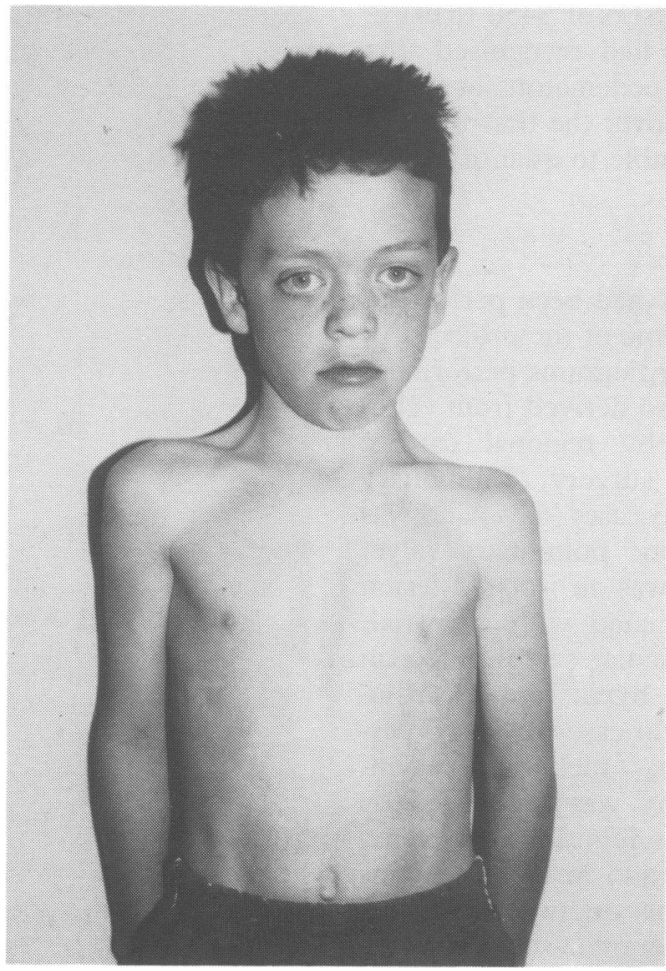

Figure 3 Child with Noonan syndrome aged 8 years.

serous otitis media. Nerve deafness requiring hearing aids had been detected in five children $(3 \%)$.

Tests of vision had been performed on 145 individuals. These were reported as abnormal in $80(55 \%)$. Because of this high figure, a cohort of 52 consecutive individuals underwent full orthoptic and ophthalmological evaluation during the study day. Strabismus was detected in $33(63 \%)$ with $16(31 \%)$ demonstrating amblyopia. Significant refractive errors (both myopia and hypermetropia) were present in 35 $(67 \%)$. Only three $(6 \%)$ of the cohort had a normal eye examination.

One hundred individuals were of school age or older. Of these, 84 individuals either were attending or had attended a normal primary or secondary school. A further five children attended a school for the physically handicapped. Only 11 children required schooling at a school for the educationally subnormal (11\%).

\section{GROWTH}

The height, weight, and head circumference data were compared with normal centiles. The data were plotted on Tanner and Whitehouse charts and the closest centile was recorded. The results are shown for height (fig 4), weight (fig 5 ), and head circumference (fig 6). Twelve of the study population were receiving biosynthetic growth hormone treatment at the time of the study, and growth data was utilised from before the treatment was commenced. Bone age (measured by the standard Greulich and Pyle method) was assessed in 37 cases. The mean (SD) delay of the bone age behind chronological age was $2 \cdot 0(1 \cdot 36)$ years.

Undescended testicles were detected in 64 out of 83 males (77\%). Puberty was delayed in both

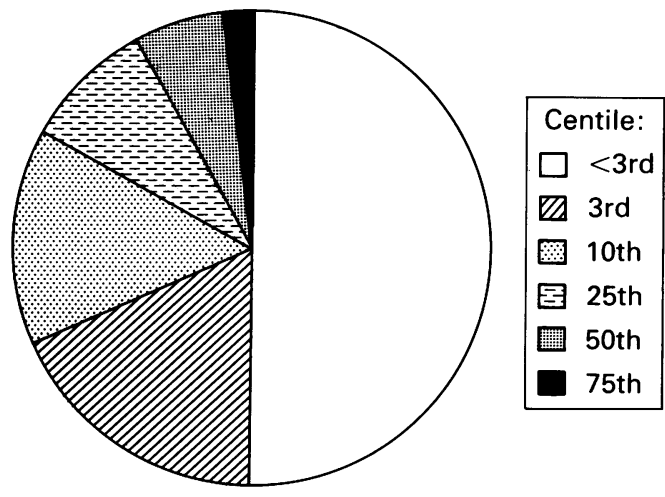

Figure 4 Height compared with normal centiles: percentage of total group.

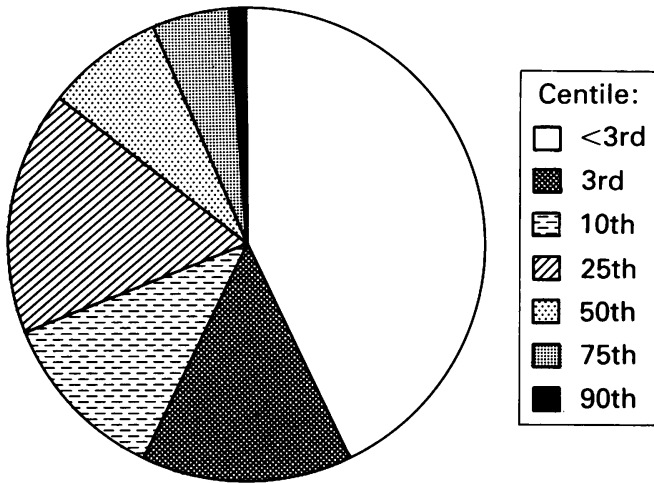

Figure 5 Weight compared with normal centiles: percentage of total group.

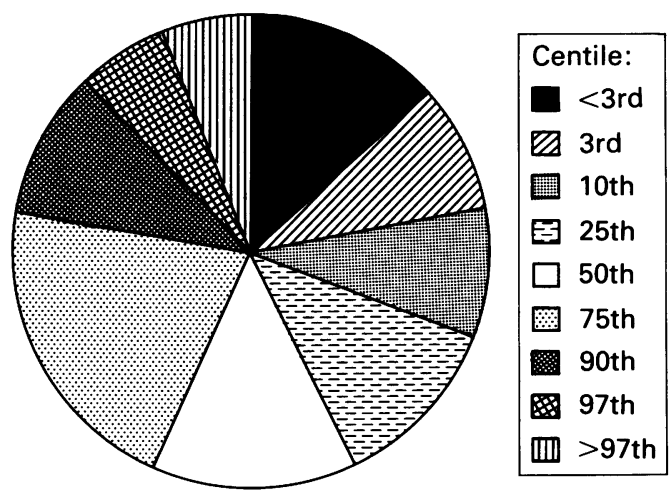

Figure 6 Head circumference compared with normal centiles: percentage of total group.

sexes. The mean (SD) age of menarche in 20 women was $14.6(1 \cdot 17)$ years. In the men puberty was reported as being significantly delayed and prolonged, but accurate data were not available retrospectively.

\section{HISTORY OF BLEEDING}

Easy bruising and bleeding were frequently reported by the study group. Bleeding histories were defined as normal, mild, moderate, or severely abnormal. Mildly abnormal bleeding was defined as easy bruising, with raised bruises $>5 \mathrm{~cm}$ in diameter occurring over the whole body after minor trauma. Moderately abnormal 
bleeding was defined as major bruising occurring after surgery (for example, orchidopexy or cardiac catherisation) or postoperative bleeding lasting for over 24 hours (for example, after dental extraction). Major abnormal bleeding was defined as a life threatening haemorrhage requiring emergency blood transfusion. A normal history was found in 63 cases $(42 \%)$. A mildly abnormal history was given by 51 patients (34\%), moderately abnormal by 32 patients $(21 \%)$, and a severe haemorrhage had occurred in five patients $(3 \%)$.

\section{ABDOMEN}

Hepatosplenomegaly unexplained by cardiac failure was a common clinical finding in the total group (26\%). This was more prominent in younger children and decreased with age. There had been no case of hepatic dysfunction associ. ated with the organomegaly. A further cohort of 45 consecutive patients subsequently underwent abdominal ultrasound examination. Significant hepatosplenomegaly was detected in 23 cases $(51 \%)$ with renal abnormalities seen in only five cases $(11 \%)$. In one child of monozygotic twins, abdominal ultrasound revealed a choledochal cyst. An ultrasound of the second twin was normal.

\section{ORTHOPAEDIC ABNORMALITIES}

The classical sternal change seen in Noonan syndrome (pectus carinatus superiorly and pectus excavatum inferiorly) was present in 144 out of 151 cases $(95 \%)$. A thoracic scoliosis was detected in 20 individuals (13\%) but in no case had this required medical or surgical treatment. Other orthopaedic abnormalities included talipes equinovarus (12\%), joint contractures $(4 \%)$, radioulnar synostosis $(2 \%)$, and cervical spine fusion $(2 \%)$.

\section{NEUROMUSCULAR ABNORMALITIES}

Significantly abnormal joint hyperextensibility was found in 75 cases (50\%). Hypotonia was also a common clinical finding. Serum creatine kinase was measured in a cohort of 35 consecutive patients. All concentrations were in the normal range for age and sex, and had a mean of $60.5 \mathrm{IU} / 1$.

Recurrent convulsions had occurred in 20 individuals $(13 \%)$. These were grand $\mathrm{mal}$ seizures in 14 cases (9\%), temporal lobe epilepsy in four cases $(3 \%)$, and febrile convulsions in two cases $(1 \cdot 5 \%)$. An unexplained peripheral neuropathy leading to distal weakness and altered sensation was detected in three adults and one child. Cervical cord compression had been excluded in all cases, and investigation had failed to reveal any cause for the neurological findings.

\section{SKIN CHANGES}

A considerable range of ectodermal changes was found. Ulerythema ophryogenes is a severe follicular keratosis previously noted to be associated with Noonan syndrome. ${ }^{8}$ It occurs predominantly on the extensor surfaces and the face, where it may lead to absent eyebrows. This particular dermatological appearance was seen in 21 cases $(14 \%)$. The hair in Noonan syndrome has previously been described as either thick and curly or thin and sparse. ${ }^{9}$ Normal hair was present in 91 cases $(60 \%)$ thick and curly hair in 44 cases $(29 \%)$, and sparse hair was present in 16 cases (11\%). The most useful skin sign was an extra prominence on the pads of all fingers and toes (fetal pads), which was present in 101 cases $(67 \%)$. Multiple lentigines (over 100 counted on the trunk) were present in five cases $(3 \%)$.

\section{OTHER ASSOCIATIONS}

Abnormalities in the lymphatic system were not common, apart from minor pedal lymphoedema. One case each was documented of lymphatic dysplasia on the lip, ${ }^{10}$ a lymphatic cyst in the neck, and pulmonary lymphangiectasia. Hypothyroidism had been documented in six cases. Antimicrosomal thyroid antibodies were measured in 23 consecutive asymptomatic cases and were positive in seven $(30 \%)$. Evidence of a further autoimmune dysfunction was provided by one case each of vasculitis, vitiligo, and anterior uveitis. Evidence of a possible immunodeficiency was suggested by frequent lower respiratory infections, and one case each of a brain abscess, bacterial endocarditis, and osteomyelitis. The differences in clinical phenotype between individuals with and without an affected first degree relative were studied using $\chi^{2}$ analysis of eight variables. No significant difference was observed between the two groups with respect to incidence of cardiac disease, developmental delay, epilepsy, or neck webbing. However, individuals without an affected parent with Noonan syndrome, did have a significantly increased incidence of short stature $(\mathrm{p}<0.01)$, skeletal abnormalities $(\mathrm{p}<0.01)$, hepatosplenomegaly $(p<0.01)$, and sparse hair $(\mathrm{p}<0.01)$.

\section{Discussion}

The incidence of Noonan syndrome in the UK population is unknown. It may be the second commonest syndrome associated with congenital heart disease after Down's syndrome. Previous reviews have suggested an incidence of between one in 1000 and one in 2000 of the population. ${ }^{67}$ Considering the frequency and importance of Noonan syndrome, surprisingly few clinical studies have been reported. A total of six complete studies have been performed, giving details on some aspects of the phenotype in 216 patients. $^{2}{ }^{11-15}$

Short stature in Noonan syndrome has been well defined on retrospective data and centile charts are available. ${ }^{16}{ }^{17}$ This study has demonstrated clearly that the growth reduction seen in Noonan syndrome is the same for both height and weight. It is interesting, however, that the head circumferences of this group were normally distributed, as macrocephaly has been reported previously. ${ }^{6}$ The high incidence of cardiac anomalies in this group may have been due to 
the continued reluctance to make a diagnosis of Noonan syndrome in the absence of congenital heart disease. Pulmonary stenosis has been the commonest cardiac abnormality reported in all previous studies, with a frequency between 25-90\%. Hypertrophic cardiomyopathy is increasingly recognised by echocardiography, and its frequency in this group (20\%) is twice that previously reported in studies using angiography. ${ }^{13} \mathrm{~A}$ further important point to be highlighted from this study is the low incidence of significant renal abnormalities in Noonan syndrome compared with Turner's syndrome. ${ }^{18}$ Musculoskeletal changes have been recognised as an important component of the syndrome. However previous reports of an association between Noonan syndrome and malignant hyperpyrexia have not been confirmed in this series. ${ }^{1920}$ Hypotonia and hyperextensibility in the younger child probably explain much of the early delay in motor milestones. Developmental outcome in the older child does not seem to confirm earlier reports of frequent mental retardation. ${ }^{2} 1112$ Hopkins-Acos and Bunker made the relevant point that speech delay may be related to hearing loss in Noonan syndrome, ${ }^{21}$ and serous otitis media was a common finding in the study group. Although specific learning disabilities have been reported in a few patients, ${ }^{22}$ more detailed studies are needed.

Clinical differences were observed between apparently familial and sporadic cases of Noonan syndrome. The apparently sporadic cases seen in this study were shorter with an increased incidence of skeletal changes, hepatosplenomegaly, and sparse hair. Only one previous smaller study had reported differences between sporadic and familial cases and had noted an increased frequency of developmental delay and short stature in the familial group. ${ }^{11}$ The most likely explanation for the clinical differences noted between the two groups in our study is selection bias, with a less severe phenotype accepted for diagnosis in familial cases. However, it is also possible that these findings may represent evidence for heterogeneity within Noonan syndrome.

\section{DIAGNOSIS}

The differential diagnosis of the child with Noonan syndrome is considerable. It is important in all cases to exclude Turner's syndrome and other chromosomal abnormalities which can mimick the Noonan syndrome phenotype, for example trisomy $8 \mathrm{p}$, trisomy 22 mosaicism, and sex chromosome rearrangements. ${ }^{24-26} \mathrm{~A}$ number of teratogens in pregnancy produce a phenotype that can overlap with Noonan syndrome and need to be specifically inquired for, particularly alcohol and primidone. ${ }^{2728}$ Other syndromes that combine facial changes with short stature or cardiac abnormalities may be confused with Noonan syndrome, for example William's syndrome or Aarskog's syndrome. Both of these syndromes are now well defined however, ${ }^{29-32}$ and recognition of the full phenotype of Noonan syndrome should readily allow accurate discrimination between them. Two other syndromes have been differentiated from Noonan syndrome based on specific ectodermal changes. The association of multiple lentigines with pulmonary stenosis and deafness led to the description of leopard syndrome, ${ }^{33}$ while an emphasis on keratotic and other skin changes led to the separation of the cardiofaciocutaneous syndrome. ${ }^{34}$ The frequent ectodermal changes seen in our study population suggests that these two syndromes cannot at present justifiably be separated from Noonan syndrome. Two previous attempts have been made to produce a scoring system that would be helpful in making the clinical diagnosis of Noonan syndrome. ${ }^{14} 35$ While both are useful research tools, neither have come to be widely used in clinical practice, and diagnosis still rests on clinical pattern recognition.

The average age at diagnosis of Noonan syndrome in this group was 9.0 years. The facial features of Noonan syndrome alter with age, ${ }^{36}$ and the easiest time to make the clinical diagnosis is in early childhood. A prompt diagnosis is important not only to allow recognition of treatable associations, but also to provide genetic counselling to the family.

1 Noonan J A, Ehmke D A. Associated non cardiac malformations in children with congenital heart disease. $\mathcal{F}$ Pediatr 1963;63:468-70.

2 Noonan J. Hypertelorism with Turner phenotype. Am $\mathcal{F}$ Dis Child 1968;116:373-80.

3 Turner HH. A syndrome of infantilism, congenital webbed neck, and cubitus valgus. Endocrinology 1938;23:566-74.

4 Ullrich $O$. Turner's syndrome and status Bonnevie Ullrich. Am $\mathcal{F}$ Hum Genet 1949;1:179-200.

5 Ford CE, Jones KW, Polani PE, de Almeida JC, Briggs JH. A sex chromosome anomaly in a case of gonadal dysgenesis (Turner's syndrome). Lancet 1959;i:711-3.

6 Mendez HMM, Opitz JM. Noonan syndrome: a review. Am $\mathcal{f}$ Med Genet 1985;21:493-506.

7 Allanson JE. Noonan syndrome. 7 Med Genet 1987;24:9-13.

8 Pierini DO, Pierini AM. Keratosis pilaris atrophicans facie ulerythema ophryogenes): a cutaneous marker in the Noonan syndrome. Br J Dermatol 1979;100:409-16.

9 Baraitser M, Patton MA. A Noonan like short stature syndrome with sparse hair. 7 Med Genet 1986;23:161-4.

10 Evans DGR, Lonsdale RN, Patton MA. Cutaneous lymphangioma and amegakaryocytic thrombocytopenia in Noonan syndrome. Clin Genet 1991;39:228-32.

11 Collins E, Turner G. The Noonan syndrome-a review of the clinical and genetic features of 27 cases. $\mathcal{F}$ Pediatr the clinical and

12 Char F, Rodriguez-Fernandez HL, Scott CI, Borgaonkar DS, Bell BB, Rowe RD. The Noonan syndrome-a clinical DS, Bell BB, Rowe RD. The Noonan syndrome-a clinical study of forty $1972 ; 8: 110-8$.

13 Nora JJ, Nora AH, Sinha AK. The Ullrich-Noonan syndrome (Turner phenotype). Am $\mathcal{F}$ Dis Child 1974;127: 48-55.

14 Duncan WJ, Fowler RS, Farkes LG, et al. A comprehensive scoring system for evaluating Noonan syndrome. Am $\mathcal{F}$ Med Genet 1981;10:37-50.

15 Sanchez Cascos A. The Noonan syndrome. Eur Heart $f$ 1983;4:223-9.

16 Ranke MB, Heidemann P, Kunpfer C, Enders H, Schmalt AA, Bierich JR. Noonan syndrome: growth and clinical manifestations in 144 cases. Eur 7 Pediatr 1988;148:22-07.

17 Witt DR, Kenna BA, Hall JG, Allanson JE. Growth curves for height in Noonan syndrome. Clin Genet 1986;30:150-3.

18 Lemli L, Smith DW. The XO syndrome: a study of the differential phenotype in 25 patients. $\mathcal{F}$ Pediatr $1963 ; 63$ : 577-87.

19 King JO, Denborough MA. Anaesthetic induced malignant hyperpyrexia in children. F Pediatr 1973;83:37-40.

20 Hunter A, Pinsky L. An evaluation of the possible association of malignant hyperpyrexia with Noonan syndrome using of malignant hyperpyrexia with Noonan syndrome using serum

21 Hopkins-Acos P, Bunker K. A child with Noonan syndrome. f Speech Hear Disord 1979;44:494-503.

22 Money J, Kalus ME Jr. Noonan syndrome-IQ and specific disabilities. Am ₹ Dis Child 1979;133:846-50.

23 Wilson $M$, Dyson A. Noonan syndrome: speech and language characteristics. F Commun Disord 1982;15:347-52.

24 Burn J, Baraitser M, Hughes D, Soldanan-Garcia P, Taylor JE. Absent right atrioventricular connection, and double inlet ventricle due to an unbalanced familial 8:13 chromosome translocation: a cautionary tale. Pediatr Cardio 1984;5:55-60

25 Wertelecki W, Breg WR, Graham IM, Linumo IK, Pinch 

SM, Sergovich FR. Trisomy 22 mosaicism syndrome and 26 Batstone PJ, Faed MJW, Jung RT, Gosden J. 45, X/46, X dic (Y) mosaicism in a phenotypic male. Arch Dis Child (Y) mosaicism

27 Spiegel PG, Peckman WM, Rich BH, Verstaag CH, Nelson V, Dudnikov M. The orthopedic aspects of the fetal alcohol syndrome. Clin Orthop 1979;139:58-63.

28 Mytre SA, Williams $R$. Teratogenic affects associated with maternal primidone therapy. $\mathcal{f}$ Pediatr 1981;99:160-2.

29 Aarskog D. A familial syndrome of short stature associated with facial dysplasia and genital abnormalities. $\mathcal{f}$ Pediat 1970;77:856-61.

30 Grier RE, Farrington FH, Kendig R, Mamones P. Autosoma dominant inheritance of the Aarskog syndrome. Am $\mathcal{F}$ Med Genet 1983;15:39-46.
31 Williams JCP, Barratt-Boyes BG, Lowe JB. Supravalvular aortic stenosis. Circulation 1961;24:1311-8.

32 Martin NDT, Snodgrass GJAI, Cohen RD. Idiopathic infantile hypercalcaemia - a continuing enigma. Arch $D$ is infantile hypercalcaem

33 Gorlin RJ, Anderson RC, Blow M. Multiple lentigines syndrome. Am f Dis Child 1969;117:652-62.

34 Reynolds JF, Neri G, Herrmann JP, et al. New multiple congenital anomalies/mental retardation syndrome with cardio-facio-cutaneous involvement-the CFC syndrome. Am $\mathcal{Y}$ Med Genet 1986;25:413-27.

35 Preus $M$. Differential diagnosis of the Williams and the Noonan syndromes. Clin Genet 1984;25:29-34.

36 Allanson JE, Hall JG, Hughes HE, Preus M, Witt RD. Noonan syndrome: the changing phenotype. Am $\mathcal{F}$ Med Genet 1985;21:507-14.

Prolonged acute crying in a young child

A diagnosis of infantile colic is usually not difficult as there is a history of recurrent crying, often at a set time of day, in an otherwise well baby. The child who presents with a first episode of excessive crying poses a different diagnostic problem. It is a fairly frequent cause of presentation to hospital casualty departments and diagnosis may be difficult. A study from the Children's Hospital in Denver, Colorado (Steven R Poole, Pediatrics 1991;88: 450-5) gives some help in tackling this problem. Over a one year period 56 children under 2 years of age were seen in the emergency department because of the recent onset of excesssive crying without fever. Babies who stopped crying before or during examination did not have a serious cause for their crying. The diagnosis was made by physical examination in 23 of the 56 children, as a result of investigations in 11 , and only at follow up in 22 . In 10 the 'diagnosis' was 'idiopathic crying' and six were thought to have infantile colic. Ten had otitis media and the rest had a wide variety of final diagnoses ranging from getting too excited at a family reunion to being bitten by a brown recluse spider. The importance of detailed and complete examination of the skin and skeletal system is emphasised as is examination of the eyes. Three children had unexpected corneal abrasions and one an ocular foreign body. Three had unexpected skeletal or intracranial trauma and one a foreign body in the throat that had escaped the notice of two doctors and was discovered by a third using a laryngoscope after the child had been noticed to wince on swallowing. Only one child proved to have a urinary infection. Investigation was dictated by clinical findings with only urine microscopy and culture being 'routine'.

The child who presents with acute unexplained crying is a good test of diagnostic mettle. Those who don't settle down in the casualty department, home, or surgery, and in whom a diagnosis is not apparent after detailed examination should be admitted to hospital for continued observation until a diagnosis is made.

ARCHIVIST 